\title{
POTENTIALITÉS ET INTÉRÊTS DE L'ÉLEVAGE LARVAIRE DE LA CREVETTE D'EAU DOUCE INDIGĖNE MACROBRACHIUM CARCINUS (L.) (PALAEMONIDAE) AUX ANTILLES FRANÇAISES.
}

\author{
F. HERMAN (1), E. FIÈVET (2), P. BOUCHER (1)
}

(1) S.I.C.A. Guadeloupéenne d'Aquaculture, Les Plaines, 97116 Pointe-Noire, France.

(2) E.S.A. C.N.R.S. n 5023 “ Écologie des Eaux Douces et des Grands Fleuves ", Université Claude Bernard Lyon I, 69622 Villeurbanne Cedex, France. (demandes de T.A.P. et correspondance)

\section{RÉSUMË}

De précédents travaux, réalisés en laboratoire, ont montré que le développement larvaire de Macrobrachium carcinus nécessitait un environnement salin. Le nombre de larves mises en élevage lors de ces essais était cependant trop faible pour envisager une production dè masse de l'espèce. Lors de l'expérience ici présentée, 46.900 larves au stade I ont été mises en élevage à une température oscillant entre 28 et $30^{\circ} \mathrm{C}$. II a été montré qu'en faisant varier la salinité, la production de masse de post-larves était possible en 45 jours, avec un taux de survie finale de: $14 ; 3 \%$ et un taux de métamorphose de $10,6 \%$. Ces résultats sont nettement inférieurs à ceux obtenus en routine avec l'espèce indonésienne Macrobrachium rosenbergii, mais attestent des possibilités d'élevage de $M$. carcinus aux Antilles françaises et ailleurs.

Mots-clés : crevette d'eau douce, Palaemonidae, Macrobrachium carcinus, élevage larvaire de masse, Antilles françaises.

\section{REARING OF NATIVE FRESHWATER PRAWN, MACROBRACHIUM CARCINUS (L.) (PALAEMONIDAE), LARVAE : ITS PROSPECTS AND INTEREST IN THE FRENCH WEST-INDIES.}

\section{ABSTRACT}

Preliminary laboratory studies demonstrated that larvae of Macrobrachium carcinus need brackish water to develop fully. Nevertheless, the number of larvae reared in these first experiments were too low to consider intensive cultivation. In this work, we started 
with 46,900 stage I larvae a mass rearing at $28-30^{\circ} \mathrm{C}$. We highlighted that post-larvae production under varying salinity conditions is possible within 45 days, with $14.3 \%$ final survival and $10.6 \%$ metamorphosis rates. These results are low in comparison with those obtained with Macrobrachium rosenbergii, but are encouraging for the cultivation of $M$. carcinus in the French West-Indies and elsewhere.

Key-words : freshwater prawn, Palaemonidae, Macrobrachium carcinus, mass larvae rearing, French West-Indies.

\section{INTRODUCTION}

Le terme de " crevette d'eau douce " est couramment utilisé pour désigner un grand nombre d'espèces de crevettes (Crustacea, Caridea) qui vivent dans les eaux douces intérieures (lacs et rivières) ainsi que dans les estuaires. La majeure partie de ces espèces appartient à la famille des Palaemonidae et plus particulièrement au genre Macrobrachium (BATE, 1868). Ce genre renferme plus de 150 espèces et présente une distribution géographique très large couvrant les régions tropicales et subtropicales du monde entier. De nombreuses espèces de crevettes d'eau douce font l'objet de pêches artisanales (HOLTHUIS, 1980), mais très peu sont élevées en aquaculture (HANSON et GOODWIN, 1977).

Le "Bouquet Pintade " (nom français F.A.O. de Macrobrachium carcinus), communément appelé “ Ouassous » en Guadeloupe et “ Z'Habitant » en Martinique (HOSTACHE, 1992), est l'espèce la plus appréciée aux Antilles. Elle est généralement pêchée dans les rivières à l'aide de nasses cylindriques appâtées à la noix de coco (GRÉGOIRE, 1991) et se vendait plus de $200 \mathrm{FF}$ le $\mathrm{Kg}$ en 1996. Aux Antilles françaises comme dans le reste des Caraïbes, les Macrobrachium sont directement menacés par une pêche peu réglementée et excessive (absence de fermeture de pêche pendant la période de reproduction et capture de toutes les classes de taille), un braconnage sans limite (utilisation de produits toxiques), la modification des cours d'eau ou encore la pollution. Peu d'études ont cependant été conduites à ce sujet et la raréfaction de M. carcinus, reconnue par les pêcheurs antillais, n'a jamais été quantifiée. Macrobrachium rosenbergii, espèce d'origine asiatique et élevée partout dans les Caraïbes, complète sans la satisfaire entièrement ( 25 tonnes produites en Guadeloupe en 1996), la forte demande locale en crevette d'eau douce (LACROIX et S.I.C.A., 1983). De grandes quantités de $M$. rosenbergii sont également importées (près de 120 tonnes en Guadeloupe pour l'année 1996 ; estimation S.I.C.A. Guadeloupéenne d'Aquaculture d'après les chiffres I.N.S.E.E.), principalement de Thailande, et sont proposées à la population entre 60 et $90 \mathrm{FF}$ le $\mathrm{Kg}$.

M. carcinus vit dans les eaux douces et saumâtres de la côte Est de l'Amérique, de la Floride au Sud du Brésil en passant par les Antilles (HOLTHUIS, 1952). Cette espèce est largement distribuée (FIĖVET, 1998) mais reste peu abondante, par exemple, au Brésil (MOREIRA et al., 1988), en Jamaïque (HART, 1961 ; CHOUDHURY, 1971a), à Sainte-Lucie (BARNISH, 1984), en Guadeloupe (GILLET, 1983 ; FIÈVET, 1995) ou encore en Martinique (DEJOUX, 1983 ; LIM et al., 1995). Les plus grands individus, pouvant mesurer jusqu'à $20 \mathrm{~cm}$ de la pointe du rostre à la pointe du telson et exceptionnellement $30 \mathrm{~cm}$ (HOLTHUIS, 1952), recherchent les portions de rivière les plus profondes (CHACE et HOBBS, 1969). INGLE et ELDRED ont noté, dès 1960, l'intérêt de $M$. carcinus pour l'aquaculture, mais le manque de connaissances sur la biologie de cette espèce a rendu son élevage difficile (MOREIRA et al., 1988). 


\section{Tableau I}

Conditions et réussites d'élevage de Macrobrachium carcinus selon différents auteurs.

$\mathrm{PL}=$ post-larves. $\boldsymbol{t}=$ mort de toutes les larves avant leur métamorphose. * Métamorphose non atteinte par toutes les larves. Durée des métamorphoses = intervalle de temps entre l'apparition de la première et la dernière PL. Taux de métamorphose $=$ nombre de PL produites $/$ nombre de larves mises en élevage. Taux de survie finale = (nombre de larves restantes + nombre de PL produites) / nombre de larves mises en élevage.

Table I

Conditions and success of Macrobrachium carcinus larvae rearing according to different sources.

PL $=$ post-larvae. $t=$ all larvae died before metamorphosis. * Metamorphosis not achieved for all larvae. Duration of metamorphoses = time interval between first and last produced PL. Metamorphosis rate = number of PL produced / initial number of larvae. Final survival rate $=$ (final number of live larvae + number of PL produced) / initial number of larvae.

\begin{tabular}{|c|c|c|c|c|c|c|c|c|c|}
\hline Auteurs & $\begin{array}{r}\text { Salinité } \\
(\%)\end{array}$ & $\begin{array}{r}\text { Effectif } \\
\text { mis } \\
\text { en éle- } \\
\text { vage }\end{array}$ & $\begin{array}{r}\text { Den- } \\
\text { sité } \\
\text { initiale } \\
\text { larves/ } \\
\text { litre) }\end{array}$ & $\begin{array}{c}\text { Durée } \\
\text { (en j) } \\
\text { d'éle- } \\
\text { vage) }\end{array}$ & $\begin{array}{l}\text { Date } \\
\text { (j) appa- } \\
\text { rition } \\
\text { pre- } \\
\text { mière } \\
\text { PL }\end{array}$ & $\begin{array}{c}\text { Durée } \\
\text { (j) des } \\
\text { méta- } \\
\text { mor- } \\
\text { phoses }\end{array}$ & $\begin{array}{c}\text { Taux } \\
(\%) \text { de } \\
\text { méta- } \\
\text { mor- } \\
\text { phose }\end{array}$ & $\begin{array}{l}\text { Nom- } \\
\text { bre de } \\
\text { PL pro- } \\
\text { duites }\end{array}$ & $\begin{array}{l}\text { Taux } \\
(\%) \text { de } \\
\text { survie } \\
\text { finale }\end{array}$ \\
\hline $\begin{array}{c}\text { LEWIS et } \\
\text { WARD (1965) }\end{array}$ & 14 & ? & $?$ & $>90$ & 90 & $?$ & $?$ & ? & ? \\
\hline \multicolumn{2}{|c|}{$\begin{array}{l}\text { CHOUDHURY } 14 \\
(1971 a)\end{array}$} & $?$ & $?$ & 66 & 56 & 10 & $?$ & ? & ? \\
\hline \multirow{6}{*}{$\begin{array}{l}\text { CHOUDHURY } \\
\text { (1971b) }\end{array}$} & $\begin{array}{r}\text { eau } \\
\text { douce }\end{array}$ & 40 & 28,5 & $t$ & $\dagger$ & $t$ & 0 & 0 & 0 \\
\hline & 7 & 40 & 28,5 & $\dagger$ & $t$ & $t$ & 0 & 0 & 0 \\
\hline & 14 & 40 & 28,5 & 60 & 59 & 1 & 2,5 & 1 & 2,5 \\
\hline & 21 & 40 & 28,5 & $t$ & $t$ & $\dagger$ & 0 & 0 & 0 \\
\hline & 28 & 40 & 28,5 & $t$ & $t$ & $t$ & 0 & 0 & 0 \\
\hline & 33 & 40 & 28,5 & $\dagger$ & $t$ & $\dagger$ & 0 & 0 & 0 \\
\hline \multirow{7}{*}{$\begin{array}{c}\text { DUGAN et al., } \\
(1975)\end{array}$} & 6 & 25 & 6,5 & $T$ & $t$ & $T$ & 0 & 0 & $\overline{0}$ \\
\hline & 8 & 25 & 6,5 & $t$ & $t$ & $\dagger$ & 0 & 0 & 0 \\
\hline & 10 & 25 & 6,5 & $\dagger$ & $t$ & $t$ & 0 & 0 & 0 \\
\hline & 12 & 25 & 6,5 & $\dagger$ & $\dagger$ & $\dagger$ & 0 & 0 & 0 \\
\hline & 14 & 25 & 6,5 & $\dagger$ & $\dagger$ & $\dagger$ & 0 & 0 & 0 \\
\hline & 16 & 25 & 6,5 & $\dagger$ & $t$ & $\dagger$ & 0 & 0 & 0 \\
\hline & $\begin{array}{l}\text { variable } \\
{[12-22]}\end{array}$ & 25 & 6,5 & $t$ & $t$ & $t$ & 0 & 0 & 0 \\
\hline $\begin{array}{c}\text { présente } \\
\text { étude }\end{array}$ & $\begin{array}{l}\text { variable } \\
\text { (Fig. 1) }\end{array}$ & 46.900 & 33,5 & *45 & 33 & $>12$ & 10,6 & 4.950 & 14,3 \\
\hline
\end{tabular}

La majorité des travaux qui ont été publiés sur $M$. carcinus traite du développement larvaire ou des effets de la température et de la salinité sur les différents stades de l'ontogenèse, ce qui permet de définir les conditions optimales d'élevage (cf. LEWIS et WARD, 1965 ; CHOUDHURY, 1971a et 1971b ; DUGAN et FRAKES, 1973 ; DUGAN et al., 1975 ; MOREIRA et al., 1988). Le développement larvaire comporte 12 stades qui ont été décrits en 1971 par CHOUDHURY (1971a), suite aux travaux de LEWIS (1961) et de LEWIS et WARD (1965). Durant sa vie larvaire, $M$. carcinus paraît demander des salinités comprises entre 12 et $20 \%$ (valeurs variables selon les auteurs). CHOUDHURY (1971a) a réussi à mener à terme l'élevage larvaire, en 56 jours, avec une salinité variant de 14 à $16 \%$ et une alimentation à base de nauplii vivantes d'Artemia salina. 
CHOUDHURY (1971b) a également montré que la croissance était plus rapide à partir du stade IV avec une salinité de $21 \%$ au lieu de $14 \%$, mais il n'a pas obtenu de métamorphose à cette salinité. DUGAN et FRAKES (1973) puis DUGAN et al. (1975) ont entrepris des expériences similaires en faisant varier ou non la salinité au cours du développement larvaire. Ils ont obtenu la meilleure survie larvaire en utilisant une salinité constante de $14 \%$, sans toutefois obtenir de post-larve (PL). Toutes ces expériences ont été conduites avec peu de larves (25 ou 40 par série) et les taux de métamorphose restaient très faibles (inférieurs à $2,5 \%$ ) (Tableau I) ; notre travail a donc consisté à entreprendre un élevage larvaire de masse de $M$. carcinus en utilisant différentes salinités pour obtenir la meilleure survie larvaire possible.

\section{MATÉRIEL ET MÉTHODE}

L'élevage de $M$. carcinus a été réalisé à l'écloserie de Pointe-Noire (S.I.C.A. Guadeloupéenne d'Aquaculture), qui produit actuellement des post-larves (PL) de $M$. rosenbergii selon la méthode dite "de l'eau claire " (AQUACOP, 1977). Les larves sont issues de deux femelles spontanément venues dans les bassins de la S.I.C.A.. Ces deux femelles ont été pêchées au mois de juillet 1993 puis mises en stabulation pendant 15 jours, avant l'introduction d'un mâle, dans un bac en béton. Après avoir pondu, elles ont été traitées avec leurs oeufs en incubation au Vert Malachite (1ppm) et au Cétavlon (1ppm). Les deux pontes, récupérées le 7 août 1993 au "stade de l'oeil ", ont été directement transférées dans un bac cylindro-conique de $1,5 \mathrm{~m}^{3}$, déjà utilisé avec succès pour l'élevage de $M$. rosenbergii. Le bac était au départ relié au circuit fermé (filtrations mécanique et biologique par un biofiltre sur graviers coralliens) d'un cycle d'élevage larvaire de $M$. rosenbergii, l'eau utilisée était ainsi initialement la même que celle destinée à l'élevage de routine de $M$. rosenbergii (salinité de $12 \%$ ). L'élevage s'effectuant ensuite à différentes salinités (Figure 1), le circuit a été ouvert et de l'eau filtrée sur sable, décantée, chlorée puis déchlorée a été utilisée.

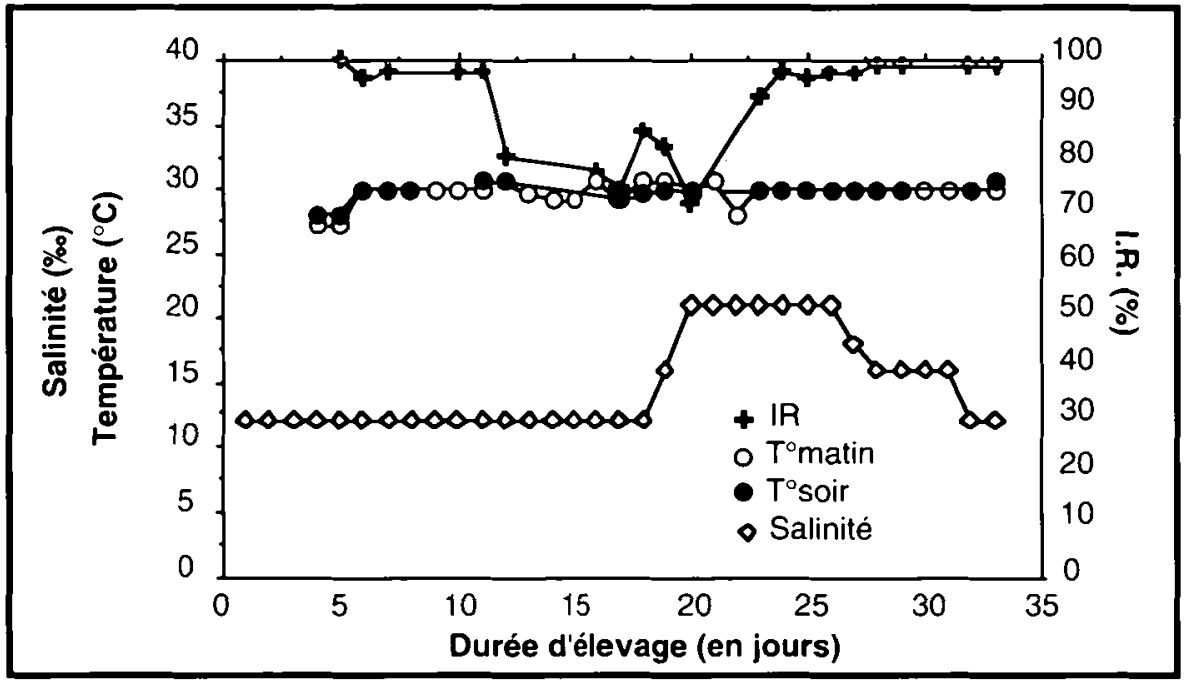

Figure 1

Variations de la salinité et de la température de l'eau au cours de l'élevage larvaire de Macrobrachium carcinus, et indice de réplétion stomacale (I.R.). Voir texte pour plus d'explications.

\section{Figure 1}

Salinity, temperature, and stomachal repletion index (I.R.) during the rearing of Macrobrachium carcinus larvae. See text for more details. 
Au 19e jour d'élevage larvaire (J19), la salinité a été augmentée progressivement jusqu'à $21 \%$ (Figure 1), valeur à laquelle, selon CHOUDHURY (1971b), la croissance des larves est maximale. A partir du stade VIII estimé (Figure 2), la salinité a été diminuée progressivement, afin d'obtenir $12 \%$ au stade $X$ et ainsi éviter une mortalité importante, comme ce fut le cas dans l'expérience de CHOUDHURY (1971b) en Jamaïque. L'expérience a été menée jusqu'à $\mathrm{J} 45$.

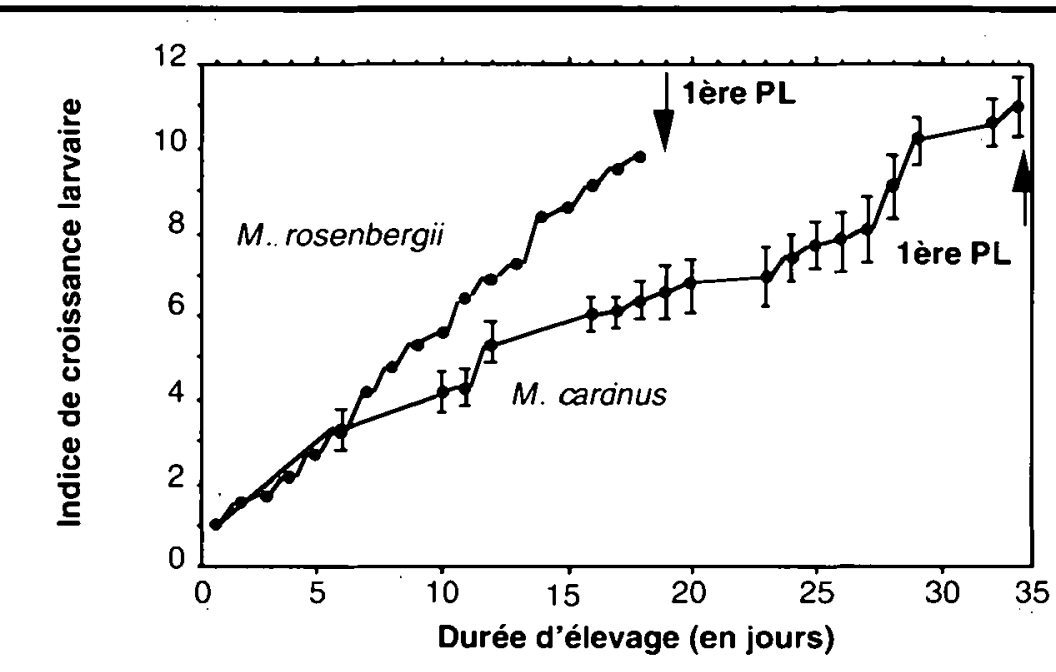

\section{Figure 2}

Indice de croissance larvaire (moyenne des $n^{\circ 8}$ des stades larvaires à une date donnée) de Macrobrachium carcinus et de Macrobrachium rosenbergii. Chaque moyenne a été calculée à partir de 30 larves. Barres d'erreur : moyenne + $/$ déviation standard. Voir texte pour plus d'explications.

\section{Figure 2}

Growth (larval stage index = mean of larval stage numbers) of Macrobrachium carcinus and Macrobrachium rosenbergii larvae. In each sample, mean values are based on 30 larvae. Error bars : mean $+/-$ standard deviation. See text for more details.

La température de l'eau a été mesurée matin (7h30) et soir (17h30) à l'aide d'un thermomètre à mercure. Elle était le plus souvent de $30^{\circ} \mathrm{C}$ et toujours comprise entre 28 et $30,5^{\circ} \mathrm{C}$ (Figure 1). S'agissant d'études préliminaires, les autres paramètres physicochimiques, notamment le $\mathrm{pH}$ et la concentration en nitrites, n'ont pas été contrôlés.

L'alimentation des larves était constituée de nauplii vivantes d'artémies (origine Utah) distribuées ad libitum et d'Acal ("Aliment calmars "), aliment artificiel alginaté préparé sur place à partir de calmars, d'oeufs, de prémix-vitaminique porcin, de vitamine $C$, d'huile de foie de morue et d'alginate. La taille des particules a été ajustée en fonction de celle des larves et a varié de 200 à $1.200 \mu$. Le niveau des rations était régulièrement ajusté grâce aux suivis des aliments non ingérés. L'Acal était proposé une fois par jour à 10 hoo et les artémies deux fois (à 7 h30 et à 13h30). 
Les indicateurs de suivi de l'élevage ont été les mêmes que ceux utilisés pour les larves de $M$. rosenbergii :

- les indices de réplétion stomacale (I.R., estimation du taux de remplissage moyen de l'estomac) ont été déterminés une heure après la première distribution d'artémies, sur un échantillon de 30 larves, par observation à la loupe binoculaire.

- l'indice de croissance (moyenne des $n^{\text {os }}$ des stades larvaires calculèe sur 30 individus) a été déterminé une fois par jour (à 8h30). Les 12 stades larvaires, reconnaissables morphologiquement, ont été déterminés à l'aide des caractères décrits par CHOUDHURY (1971a). II s'agissait notamment de la forme ou du stade de développement des yeux, des épines épigastriques, du telson et des divers appendices.

- les restes alimentaires ont été examinés et mesurés chaque jour.

- les morts apparents ont été siphonnés puis comptés une fois par jour en même temps que les restes d'Acal.

Les paramètres d'élevage de l'étude ici présentée et de travaux précédents sont indiqués dans le Tableau I.

\section{RÉSULTATS ET DISCUSSION}

\section{Performances d'élevage}

46.900 larves au stade I de leur développement ont été initialement mises en élevage. Le taux de métamorphose $(10,6 \%)$ est très supérieur à ceux obtenus par CHOUDHURY (1971b) (Tableau I). II est cependant bien moindre que ceux obtenus en routine à l'écloserie de Pointe-Noire avec $M$. rosenbergii $(75-80 \%)$. L'arrêt de l'élevage avant l'obtention des dernières post-larves (1.750 larves encore vivantes et non métamorphosées) est inhérent aux exigences d'élevage de masse, à savoir l'obtention du plus grand nombre de post-larves $(\mathrm{PL})$ en un minimum de temps. Il serait utile de déterminer le meilleur compromis (financier) possible entre ces deux facteurs. 33 jours ont été nécessaires pour obtenir la première $\mathrm{PL}$, ce qui est relativement rapide par rapport aux résultats précédents (Tableau I). Le développement larvaire complet (obtention d'une PL) le plus rapide est de 33 jours (Figure 2), alors qu'il n'est que de 22 jours pour Macrobrachium acanthurus (espèce présente aux Antilles) (DUGAN et FRAKES, 1973) et de 18 jours pour $M$. rosenbergii à l'écloserie de Pointe-Noire (Figure 2). Le développement larvaire de $M$. rosenbergii est plus rapide que celui de $M$. carcinus dès le $6^{\mathrm{e}}$ jour d'élevage (Figure 2).

Les morts récoltés par siphonnage (Figure 3) ne représentent qu'une infime partie de la mortalité réelle $(23,4 \%)$, ce qui laisse supposer un cannibalisme intense. Ce cannibalisme a pu être particulièrement important durant les 9 premiers jours et/ou après le $15^{\mathrm{e}}$ jour d'élevage ; périodes pendant lesquelles aucun mort journalier n'a été le plus souvent comptabilisé (Figure 3), contrairement entre J10 et J15 (921 morts en 6 jours). La grande différence de taille observée (du simple au double pour les larves en fin d'élevage) favorise par ailleurs un tel cannibalisme. Des particules alimentaires (Acal ou chair de poissons finement hachée) peuvent être distribuées afin que les larves s'y fixent et évitent ainsi de s'endommager entre elles. Ces particules (Acal, dans notre cas) sont cependant peu attirantes et ne limitent pas le cannibalisme de façon significative. Une autre façon de limiter le cannibalisme serait de réduire la densité des larves mises en élevage. Il serait néanmoins utile de déterminer ultérieurement les différentes causes exactes de mortalité aux différents stades. 


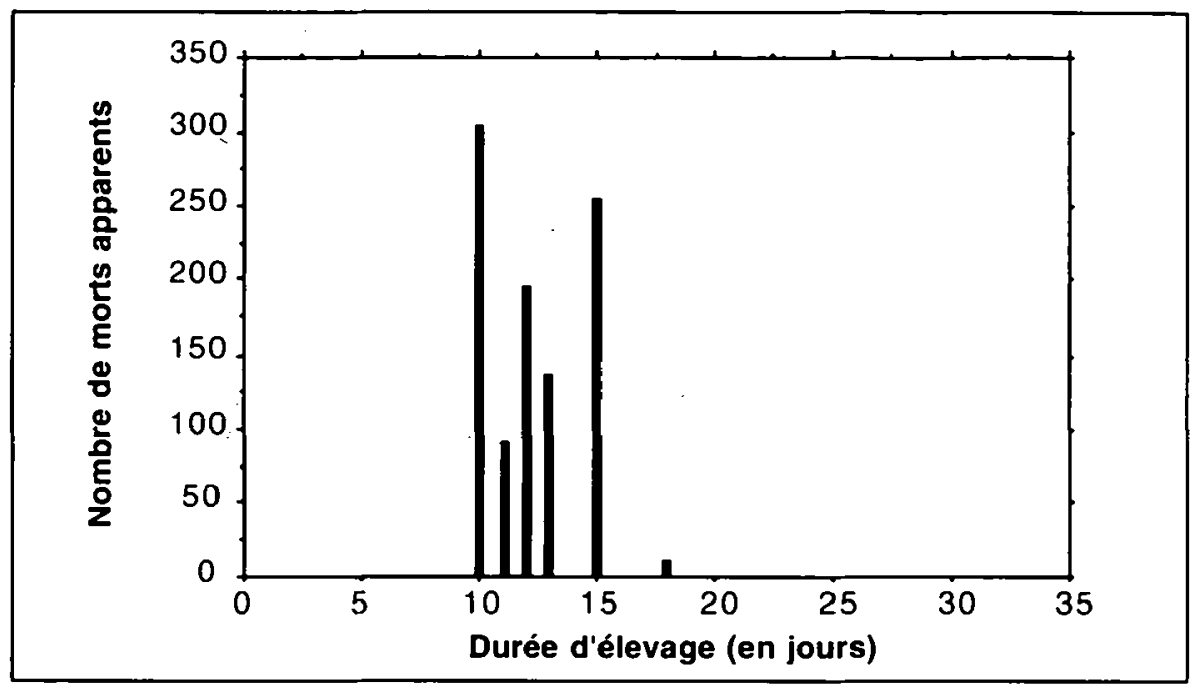

Figure 3

Nombre apparent de larves de Macrobrachium carcinus mortes chaque jour.

Figure 3

Apparent number of Macrobrachium carcinus larvae that died each day.

\section{Paramètres d'élevage}

La qualité de l'eau n'a pas été mise en cause puisque les élevages larvaires de $M$. rosenbergii conduits en parallèle ont abouti à des résultats habituels de survie. $M$. carcinus peut cependant être plus sensible à la qualité de l'eau, notamment à l'accumulation toxique d'ammoniaque. Un telle accumulation est cependant peu probable ; la concentration en ammoniaque n'a en effet jamais excédé $0,02 \mathrm{ppm}$ et celle en nitrites $0,1 \mathrm{ppm}$ lors des précédents contrôles de la qualité de l'eau utilisée pour l'élevage larvaire de $M$. rosenbergii en circuit fermé, et les risques étaient limités à partir de J18 du fait du renouvellement de l'eau. La température influence fortement la durée du développement larvaire. Le développement larvaire de $M$. rosenbergii, par exemple, mené à $28^{\circ} \mathrm{C}$ dure en moyenne une semaine de plus qu'à $30^{\circ} \mathrm{C}$ (S.I.C.A. Guadeloupéenne d'Aquaculture, données non publiées). La température de l'eau a oscillé entre 24 à $28^{\circ} \mathrm{C}$ lors des expériences de CHOUDHURY (1971a et b), alors qu'elle était plus constante et élevée dans notre essai d'élevage de masse $\left(30^{\circ} \mathrm{C}\right.$ le plus souvent, sauf en début d'expérimentation, Figure 1). Le passage entre stades larvaires s'est accéléré quand la salinité est passée de 12 à $21 \%$ et l'indice de réplétion stomacale (I.R.) est apparu meilleur à cette salinité (Figure 1). Puisque $M$. carcinus nécessite de l'eau saumâtre lors de sa vie larvaire, le premier facteur à avoir été étudié est le taux de salinité. Les exigences en sel ne sont probablement pas les mêmes d'un stade à l'autre (CHOUDHURY, 1971b), mais restent difficiles à évaluer. Les différences d'optima de salinité constatées par les auteurs (14-17,5\% pour CHOUDHURY (1971b), $12 \%$ pour DUGAN et al. (1975) et 16-18 \% pour la S.I.C.A. Guadeloupéenne d'Aquaculture (données non publiées)) démontrent la possibilité d'interactions entre la salinité et les autres paramètres d'élevage.

L'alimentation pose un problème majeur et l'échec de nombreuses tentatives d'élevage lui est probablement lié (e.g., HOSTACHE, 1977). Les premiers stades larvaires, qui ne sont pas détritivores, nécessitent une alimentation vivante à leur taille (zooplancton) (CHOUDHURY, 1971b). Des larves d'artémies, facilement cultivables, sont 
généralement utilisées et conduisent à de bons résultats avec $M$. rosenbergii, mais ne sont pas forcément bien adaptées à tous les stades larvaires chez $M$. carcinus. La grosseur des particules alimentaires (Acal) a été ajustée à la taille des larves lors de l'élevage de $M$. carcinus ; ces particules ont cependant été très peu ou pas du tout ingérées. La première mue peut être atteinte sans alimentation grâce aux réserves vitellines (CHOUDHURY, 1971b) et la seconde ou les suivantes, grâce au cannibalisme. La consommation alimentaire, compte tenu de la faible survie, est évidemment très supérieure à celle obtenue pour $M$. rosenbergii. En effet, en extrapolant les résultats, la production d'un million de post-larves de $M$. carcinus nécessiterait la consommation de $117 \mathrm{Kg}$ d'oeufs d'artémies et de $70 \mathrm{Kg}$ d'Acal contre respectivement 22 et $35 \mathrm{Kg}$ pour M. rosenbergii.

\section{Perspectives et intérêts}

La date d'apparition de la première $\mathrm{PL}$, mais aussi le taux de survie finale et le taux de métamorphose, sont clairement liés aux conditions d'élevage. L'effet de paramètres facilement contrôlables comme la température, la concentration en divers ions, le $\mathrm{pH}$ (paramètres physico-chimiques) ou la luminosité, est important à connaître afin de déterminer les conditions optimales d'élevage. Dans la mesure où la salinité semble avoir un rôle primordial, il serait utile de réaliser des recherches de choix de salinité des larves aux différents stades. II serait également important de tester d'autres aliments, comme par exemple des copépodes ou des rotifères vivants. La mise au point d'un protocole d'élevage bien rodé de $M$. carcinus est tout à fait envisageable, mais nécessite davantage d'expériences. L'objectif de $30 \%$ de survie finale après 45 jours paraît réalisable.

La production de PL de $M$. carcinus en 45 jours avec un taux de métamorphose proche des $10 \%$ est un résultat déjà satisfaisant pour envisager des expériences d'élevage de PL ou pour assurer des lâchers de repeuplement en milieu naturel. Certaines des 4.950 larves obtenues lors de cet élevage seront mises en bassin de grossissement. Cette seconde phase renseignera sur la croissance et le taux de survie de $M$. carcinus en bassin d'élevage, et donc sur les possibilités d'aquaculture. Le suivi d'une mono-cohorte permettra de vérifier si la distribution en classes de taille devient polymodale après un certain temps comme pour $M$. rosenbergii, chez qui des différenciations sexuelles et morphologiques (phénomènes de dominance) apparaissent (BARKI et al., 1991).

Des lâchers en rivières sont également envisageables. II serait néanmoins utile de vérifier, dans un premier temps, si de tels lâchers permettraient un réel renforcement des populations. Des études complémentaires en milieu naturel comme celles de LEWIS et al. (1966) et de VALENTI et al. (1986) sont également nécessaires pour augmenter la somme des connaissances biologiques sur $M$. carcinus. Différentes techniques d'échantillonnage (FIĖVET et al., 1996 ; FIÉVET et al., 1998) et de marquage sont actuellement en cours d'évaluation pour suivre les populations de $M$. carcinus et les peuplements de crevettes en général, dans les rivières antillaises.

\section{REMERCIEMENTS}

Ce travail a bénéficié d'un soutien financier du Fond d'Intervention de l'Agriculture sollicité par le Parc National de la Guadeloupe. Nous remercions le Professeur L.R. d'ABRAMO (Univ. du Mississippi), pour ses conseils préliminaires. L'élevage a pu être mené à bien grâce au recrutement de deux stagiaires : Y.M. MORVAN (Univ. de Caen) et E. SHLESSER (Univ. de Montpellier), que nous remercions ici vivement. Nos remerciements vont également à $H$. TACHET et E. PATTEE (Univ. Lyon I) et à un examinateur anonyme, pour leurs conseils de relecture. 


\section{BIBLIOGRAPHIE}

AQUACOP, 1977. Macrobrachium rosenbergii (de Man) culture in Polynesia : progress in developing a mass intensive larval rearing technique in clear water. Proc. World Maricult. Soc., 8, 311-326.

BARKI A., KARPLUS I., GOREN M., 1991. Morphotype related dominance hierarchies in males of Macrobrachium rosenbergii (Crustacea; ..Palaemonidae):..Behaviour; - 1.17; 145-160.

BARNISH G., 1984. The freshwater shrimps of Saint Lucia, West Indies (Decapoda, Natantia). Crustaceana, 47, 314-320.

BATE C.S., 1868. On a new genus, with four new species, of freshwater prawns. Proc. Zool. Soc. Lond., 1868, 363-368.

CHACE F.A. JR., HOBBS H.H. JR., 1969. The freshwater and terrestrial decapod crustaceans of the West Indies with special reference to Dominica. U.S. Nat. Mus. Bull., 292, 1-258.

CHOUDHURY P.C., 1971a. Complete larval development of the palaemonid shrimp Macrobrachium carcinus (L.), reared in the laboratory (Decapoda, Palaemonidae). Crustaceana, 20, 51-69.

CHOUDHURY P.C., 1971b. Responses of larval Macrobrachium carcinus (L.) to variations in salinity and diet (Decapoda, Palaemonidae). Crustaceana, 20, 113-120.

DEJOUX C., 1983. Mission hydrobiologique en Martinique. Rap. O.R.S.T.O.M., Fort-deFrance, 75 p., rapport non publié.

DUGAN C.C., FRAKES T.A., 1973. Culture of brackish-freshwater shrimp, Macrobrachium acanthurus, M. carcinus and M. ohione. Proc. World Maricult. Soc., 3, 185-191.

DUGAN C.C., HAGOOD R.W., FRAKES A.T., 1975. Development of spawning and mass larval rearing techniques for brackish-freshwater shrimps of the genus Macrobrachium (Decapoda, Palaemonidae). Florida Dept. nat. Resour., Marine Res. Publ., 12, 1-28.

FIÈVET E., 1995. Comparaison de deux méthodes d'échantillonnage quantitatif et étude de la co-structure faune-milieu de quatre cours d'eau de Guadeloupe. Convention d'étude Parc National de la Guadeloupe/C.N.R.S. Rap. de D.E.A., Univ. Lyon I, Villeurbanne, 35 p. + annexes, rapport non publié.

FIĖVET E., 1998. Distribution et capacités d'expansion des crevettes d'eau douce de la région caraïbe : exemple des genres Macrobrachium et Atya (Crustacea : Caridea). Biogeographica, 74, 1-22.

FIĖVET E., TITO DE MORAIS L., TITO DE MORAIS A., 1996. Quantitative sampling of freshwater shrimps : comparison of two electrofishing procedures in a Caribbean stream. Arch. Hydrobiol., 138, 273-287.

FIĖVET E., BONNET-ARNAUD P., MÉNARD C., TACHET H., 1998. Étude de la migration amont des crevettes d'eau douce au niveau des obstacles artificiels : implications pour les dispositifs de franchissement. Convention d'étude D.I.R.E.N. Guadeloupe/Parc National de la Guadeloupe/C.N.R.S. Laboratoire d'Écologie des Eaux Douces et des Grands Fleuves, Univ. Lyon I, 47 p., rapport non publié.

GILLET C., 1983. Les peuplements de poissons et de crevettes des rivières de la Guadeloupe : quelques données sur la biologie, la reproduction, la répartition des espèces. Rev. Hydrobiol. Trop., 16, 327-340.

GRÉGOIRE D., 1991. Étude socio-économique de la pêche en rivière dans la zone périphérique du Parc National de la Guadeloupe. Rap. Univ. Bordeaux I/Parc National de la Guadeloupe, 40 p. + annexes, rapport non publié.

HANSON J.A., GOODWIN H.L. (eds.), 1977. Shrimp and prawn farming in the western hemisphere. Second workshop on the culture of Macrobrachium spp. Dowden, Hutchinson and Ross Inc., Stroudsburg, Pennsylvania, 439 p. 
HART C.W. JR., 1961. The freshwater shrimps (Atyidae and Palaemonidae) of Jamaica, W. I. With a discussion of their relation to the ancient geography of the western Caribbean area. Proc. Acad. Nat. Sci., Phila. 113, 61-80.

HOLTHUIS L.B., 1952. A general revision of the Palaemonidae (Crustacea, Decapoda) of the Americas. II-The subfamily Palaemoninae. Allan Hancock Found. Occ. Pap., 12, 1-396.

HOLTHUIS L.B., 1980. Shrimps and prawns of the world, an annotated catalogue of species of interest to fisheries. F.A.O. Fish. Synop., 125, 1-271.

HOSTACHE G., 1977. Contribution à l'étude des crevettes d'eau douce de la Guadeloupe. Rap. I.N.R.A. Guadeloupe, 120 p., rapport non publié.

HOSTACHE G., 1992. La vie dans les eaux douces de la Guadeloupe ; poissons et crustacés. I.N.R.A. et Parc National de la Guadeloupe (eds.), 84 p.

INGLE R., ELDRED B., 1960. Notes on the artificial cultivation of fresh water shrimp. West Indies Fish. Bull., 1960(4), 1-5.

LACROIX D., S.I.C.A., 1983. L'aquaculture du Macrobrachium rosenbergii aux Antilles françaises. Actes de colloques "Bases biologiques de l'aquaculture ", Montpellier, 12-16 déc. 1983, IFREMER, 1, 263-275.

LEWIS J.B., 1961. Preliminary experiments on the rearing of the fresh water shrimp Macrobrachium carcinus (L.). Proc. Gulf Caribb. Fish. Inst., 14, 199-201.

LEWIS J.B., WARD J., 1965. Developmental stages of the palaemonid shrimp Macrobrachium carcinus (Linnaeus, 1758). Crustaceana, 9, 137-148.

LEWIS J.B., WARD J., McIVER A., 1966. The breeding cycle, growth and food of the fresh water shrimp Macrobrachium carcinus (Linnaeus). Crustaceana, 10, 48-52.

LIM P., DAUBA F., DAUTA A., BOSCA C., 1995. Étude faunistique des rivières martiniquaises. Convention I.N.P. E.N.S.A.T./U.P.S./D.I.R.E.N. Martinique, 116 p. + annexes, Institut National Polytechnique, Toulouse, rapport non publié.

MOREIRA G.S., NGAN P.V., MOREIRA P.S., SHUMWAY S.E., 1988. The effect of salinity on the osmo-ionic regulation of Macrobrachium carcinus (Linnaeus). Comp. Biochem. Physiol., 91A, 105-108.

VALENTI W.C., MELLO J.T.C.D., LOBÃO V.L., 1986. Dinâmica de reprodução do Macrobrachium acanthurus (Wiegmann, 1836) e Macrobrachium carcinus (Linnaeus, 1758) do rio Ribeira de Iguape (Crustacea, Decapoda, Palaemonidae). Ciência e Cultura, São Paulo, 38, 1256-1262. 